\title{
Why Directionality Is an Important Light Factor for Human Health to Consider in Lighting Design?
}

\author{
Parisa Khademagha, ${ }^{1,2, *}$, Myriam Aries ${ }^{\mathbf{1 , 3}}$, Alexander Rosemann",2, Evert Van \\ Loenen ${ }^{1,2,4}$ \\ ${ }^{1}$ Eindhoven University of Technology, Department of the Built Environment, Building \\ Lighting Group, Eindhoven, the Netherlands \\ ${ }^{2}$ Intelligent Lighting Institute, Eindhoven University of Technology, Eindhoven, the \\ Netherlands \\ ${ }^{3}$ Jönköping University, School of Engineering, Department of Construction Engineering and \\ Lighting Science, Jönköping, Sweden \\ ${ }^{4}$ Philips Research, Eindhoven, the Netherlands
}

Received 03 December 2016, Accepted 19 December 2016

\begin{abstract}
Both image-forming and non-image-forming effects of radiation require proper attention in lighting design that aims at meeting human vision and health requirements. Intrinsically Photosensitive Retinal Ganglion Cells (ipRGCs) appear to play an essential role in stimulation of the non-image forming effects and thus human health and well-being. There are indications that radiation incident contributes to the magnitude of these effects. This review summarizes current studies on humans and animals related to radiation directionality as well as the spatial distribution of ipRGCs on the retina. New insights can facilitate and optimize the incorporation of radiation directionality in building lighting design.
\end{abstract}

Keywords: Non-image-forming light effects, ipRGCs, Melanopsin, Incidence

\section{Introduction}

Ocular radiation exposure is not only important for its image-forming (IF) effects enabling humans to perform visual tasks, but also for its short-term (acute) and long-term (e.g., circadian) non-image-forming (NIF) effects influencing human health and well-being.

A non-rod, non-cone photoreceptor type in the human eye called "intrinsically photosensitive Retinal Ganglion Cell” (ipRGC) is primarily responsible for stimulation of the NIF effects [1,2]. These melanopsin-expressing photoreceptors constitute a small population of the Retinal Ganglion Cells (RGCs). Since their sensitivity is different than the one of the image-forming system, these effects cannot be directly related to photometric quantities. In this paper the authors therefore refer to radiation instead of light. The ipRGCs transduce radiation received directly and via the rods and cones $[3,4]$ to neural signals which are transmitted to different parts of the brain, e.g., the Supra-Chiasmatic Nuclei (SCN). The SCN regulates a set of endogenous physiological and behavioral (e.g., circadian) rhythms through its connection to the central nervous system. Sleep-wake cycle, melatonin (sleep hormone) secretion, and alertness pattern are examples of circadian rhythms. Interruption of the

\footnotetext{
* Corresponding Author: P. Khademagha, Tel.: +31 40-247 2302

E-mail address: p.khademagha@tue.nl
} 
entrainment of circadian rhythms with the 24h light/dark cycle can play a role in mood disorders [5], cardiovascular disease [6], and even cancer [7,8]. Radiation has, in addition to the long-term effects, short-term effects e.g. secretion of melatonin hormone, pupillary reflex, and objective/ subjective alertness.

Light factors stimulating the NIF effects in humans show two distinct characteristics (luminous and temporal) [9]. The implementation of the three luminous light factors 'quantity', 'spectrum', and 'directionality' in building lighting design has been investigated. Literature on the impact of radiation directionality with regards to NIF effects on humans is rather limited [9-13]. Nevertheless, studies have shown that the radiation incident to the human eye plays an important role in the magnitude of the NIF effects. It has been proposed that the influence of radiation directionality on NIF effects might be due to differences in either sensitivity of the ipRGCs in different parts of the retina, or their spatial distribution throughout the retina [12,13]. In building lighting design, directionality can be impacted through a number of ways for both daylighting and electric lighting. However, limited number of human studies is a barrier on the ways of gauging which of the proposed possibilities with regards to the population and sensitivity of ipRGCs are more plausible. Therefore, to better understand the influence of radiation incident on NIF effects in this paper we have reviewed both human and animal studies in which radiation directionality or distribution of ipRGCs on retina have been investigated.

\section{Method}

Search on scientific literature was executed across three literature databases: Science Direct, PubMed, and Scopus. The search in PubMed was based on the article's title and abstract and in Scopus and Science Direct based on article title/abstract/keywords. Results were limited to 'journal articles' in the 'English language'. Moreover, the subject area was limited to 'neuroscience, agriculture and biological sciences, and biochemistry, genetics and molecular biology' in Scopus, 'biochemistry, genetics and molecular biology, neuroscience, and psychology' in Science Direct, and 'MEDLINE' in PubMed. Table 1 shows the exact search terms used, the number of hits, and eligible papers included in the review per database. Eligibility of the papers was assessed by analyzing their abstracts. Papers were eligible for inclusion if they addressed radiation directionality or the spatial distribution of the ipRGCs in retinas. In addition to the resulting eligible papers, relevant references from these papers were also retrieved and checked for their eligibility. Human studies identified in previously published review [9] have also been included.

Table 1. Search term, hits and number of included papers per databases (Date of last search 2016-11-14)

\begin{tabular}{|c|c|c|c|}
\hline Database & Exact search terms & Hits & $\begin{array}{l}\text { Eligible after } \\
\text { pre-selection }\end{array}$ \\
\hline ScienceDirect & $\begin{array}{l}\text { TITLE-ABSTR-KEY(ipRGC OR melanopsin) and TITLE-ABSTR-KEY(spatial distribution } \\
\text { OR density OR population OR directionality OR visual field OR retinal field OR nasal OR } \\
\text { superior) AND LIMIT-TO(contenttype, "JL,BS","Journal"). }\end{array}$ & 3 & 3 \\
\hline PubMed & $\begin{array}{l}\text { (ipRGC[Title/Abstract] OR melanopsin[Title/Abstract]) AND (spatial } \\
\text { distribution[Title/Abstract] OR density[Title/Abstract] OR population[Title/Abstract] OR } \\
\text { directionality[Title/Abstract] OR visual field[Title/Abstract] OR retinal field[Title/Abstract] } \\
\text { OR nasal[Title/Abstract] OR superior[Title/Abstract]) AND (Journal Article[ptyp] AND } \\
\text { English[lang]) AND (Journal Article[ptyp] AND English[lang] AND medline[sb]) }\end{array}$ & 92 & 29 \\
\hline Scopus & $\begin{array}{l}\text { TITLE-ABS-KEY ((ipRGC OR melanopsin) AND (spatial distribution OR density OR } \\
\text { population OR directionality OR visual field OR retinal field OR nasal OR superior)) AND } \\
\text { DOCTYPE (ar) AND (LIMIT-TO (SUBJAREA, "NEUR") OR LIMIT-TO (SUBJAREA, } \\
\text { "BIOC") OR LIMIT-TO (SUBJAREA, "AGRI")) AND (LIMIT-TO (LANGUAGE, } \\
\text { "English")) }\end{array}$ & 12 & 6 \\
\hline
\end{tabular}

\section{Results}

A throughout abstract-based selection showed that 30 unique paper were eligible for further analysis in addition to the four human studies. Except one pre-selected study in Scopus, other eligible studies found in Scopus and ScienceDirect were also found in PubMed. Two distinct approaches were used in laboratory experiments to 
investigate the spatial distribution of ipRGCs in the retina: 1) in situ experiments with human subjects, 2) in vitro experiments using isolated animal retinas. Four different areas in the retina have been studied: inferior or vertal, superior or dorsal, nasal, and temporal areas.

In experiments with human subjects, the suppression of the hormone melatonin (the hormone that regulates the sleep-awake cycle) has often been chosen as a biomarker for NIF effects of luminous radiation. Four studies have investigated the effect of radiation directionality on human melatonin suppression [10-13]. The method, light source, and experimental conditions varied in every study. For instance, while Lasko et al. [11] reported to have used an illuminance of $500 \mathrm{~lx}$ on the superior and inferior retina and disregarded the full retinal radiation exposure, Glickman et al. [12] chose to work with full retinal exposure of $100 \mathrm{~lx}$ and $200 \mathrm{~lx}$ with similar retinal photon flux in addition to superior and inferior retinal exposure of 200 lx. Moreover, in the Glickman-study retinal exposures were fully controlled with the help of a helmet with shields whereas in the Lasko-study retinal exposures were distinguished simply by moving the location of the light source from the upper to the lower part of the view gaze. In both, the study of Visser et al. [10] and the Rüger et al. [13] study , a helmet was designed for controlling only the nasal and temporal retinal exposure. For the inferior and superior radiation exposure, they relied on the lens properties of the cornea.

Aside from methodological differences in the aforementioned studies, melatonin suppression was higher when the inferior retina was illuminated compared to the superior retina [10-12]. The difference has reached statistical significance in the Glickman and Lasko studies [11,12] $(\mathrm{p}<0.05)$. In addition, a significant effectiveness of illuminating the nasal retina compared to temporal retina $(\mathrm{p}<0.05)$ in melatonin suppression was observed in the Visser and the Rüger studies [10,13]. These results suggested that the inferior and nasal retinal area are either (i) more sensitive toward radiation stimuli when the NIF effects are concerned, or (ii) contain a higher density of the ipRGCs. In vitro studies on the human retina could facilitate further investigation in order to find which one of the proposed reasons is more plausible. However, because of the barriers on the way of acquiring good-quality retinal tissue, in vitro studies on human retina are rare and none could achieve adequately staining (detecting with a cell-tracer) of the photoreceptors [14].

In animals, however, ipRGCs and their architecture have been the topic of several investigations. Their classifications, morphological, and physiological characteristics have been reviewed from different point of views [15-19]. Not all ipRGCs are in one retinal layer [16,20,21]. A distinction has been made on whether they were placed in the ganglion cell layer (known as orthotopic or normal placed) or in the inner nuclear layer (known as displaced). Moreover, five subtypes (M1-M5) of ipRGCs have been identified each with different soma size and dendrites stratifications [16,17,19]. Every subtypes projects to specific retino-recipient brain structures or central targets, e.g., SCN, olivary pretectal nucleus (OPN), and dorsal lateral geniculate nucleus (dLGN). This is particularly important as some of these central targets, e.g. dLGN, are responsible for the IF effects of radiation whereas others, e.g. SCN, are in charge of the NIF radiation effects [17,22-24]. Some central targets are innervated by more than one subtype of ipRGCs. For instance, it appears that M1 and M2 subtypes both innervate the SCN (biological clock) with M1 subtype being dominant [23].

Fifteen studies have investigated the spatial distribution of ipRGCs in animal retinas [2,20,21,24-37]. Most of the experiments were carried out using different rat species e.g. wildtype, albino, and pigmented [21,25,27-35] while only a few ones used primate [24,26] and cat [37]. To identify the location and population of the ipRGCs, a cell-tracer was injected into different central targets. To determine the spatial distribution of the stained ipRGCs, confocal microscopic pictures were taken of different retinal areas. These images were merged together for the quantitative analysis. For cell-counting, an automated neighbor mapping method was introduced by GalindoRomeo et al. [20] and validated by a manual counting method for adult albino rats. In rats, the number of ipRGCs appeared to increase along with the growth in age [28,29,38].

Despite of methodological differences in the reviewed animal studies, ipRGCs were found to be denser in the superior (dorsal) and temporal retinal areas [21,24,25,27-32,34,35]. Among all these studies [2,20,21,24-36] only three have considered different subtypes of ipRGCs in their analysis [29,30,33]. Whereas Hughes et al. [30] investigated the whole-mount retina, Esquiva et al. [29] and Jeong et al. [33] studied only a part of retina. They all have reported a non-homogenous distribution of different sub-types. Hughes et al. [30] reported a higher density of M1 and M2 subtypes in superior retina. A fairly uniform distribution of the ipRGCs was reported by Moirn et al. [25], Semo et al. [37], and by Hughes et al. [30] when all subtypes of ipRGCs have been taken into account . In addition, we should keep in mind that ipRGCs receive radiation stimuli not only intrinsically but also via the classical photoreceptors [3,30]. Hughes et al. [30] have demonstrated the influence of both rods and cones on the spectral tuning of different subtypes of ipRGCs. 


\section{Discussion and conclusion: Implementation of radiation directionality in building lighting design}

Literature on the influence of radiation directionality, especially on human subjects, is rather limited. Human studies show that inferior-nasal retinal areas are significantly more effective in stimulation of the NIF effects of radiation using melatonin as biomarker. Whether these findings are due to a higher density of ipRGCs in inferiornasal areas or a higher sensitivity of the photoreceptors in these areas is still unknown. Animal studies, however, show that ipRGCs are more densely packed in the superior-temporal retinal area. Differences in suggested spatial distribution of ipRGCs in human and animals can be attributed to either anatomical differences in their retinas or to downstream processes in ipRGCs or biological rhythms.

Further investigation of the classifications, morphological and physiological characteristics of ipRGCs in humans will be required to reason such differences in findings between animal and human studies. Although these two types of study groups suggest different spatial distributions for ipRGCs in the retina, both reveal (suggest [38]) a non-homogenous distribution for these photoreceptors and thus highlight the importance of radiation directionality on human health.

For the design of lighting in buildings, this translates into the fact that one can positively influence the occupant's health and well-being by choosing the right position for daylight openings and luminaires depending on the magnitude of the influence one is looking for. In addition, it enables different stakeholders in the (day)lighting industry to design lighting that supports both human vision and health.

\section{Acknowledgement}

This research was performed within the framework of the strategic joint research program on Intelligent Lighting Institute between TU/e and Royal Philips N.V.

\section{References}

[1] Berson, D. M., Dunn, F. A., \& Takao, M. (2002). Phototransduction by retinal ganglion cells that set the circadian clock. Science, 295(5557), 1070-1073.

[2] Hattar, S., Liao, H. W., Takao, M., Berson, D. M., \& Yau, K. W. (2002). Melanopsin-containing retinal ganglion cells: architecture, projections, and intrinsic photosensitivity. Science, 295(5557), 1065-1070.

[3] Gooley, J. J., Mien, I. H., Hilaire, M. A. S., Yeo, S. C., Chua, E. C. P., van Reen, E., ... \& Lockley, S. W. (2012). Melanopsin and rod-cone photoreceptors play different roles in mediating pupillary light responses during exposure to continuous light in humans. Journal of Neuroscience, 32(41), 14242-14253.

[4] Wong, K. Y., Dunn, F. A., Graham, D. M., \& Berson, D. M. (2007). Synaptic influences on rat ganglion-cell photoreceptors. The Journal of physiology, 582(1), 279-296.

[5] Germain, A., \& Kupfer, D. J. (2008). Circadian rhythm disturbances in depression. Human Psychopharmacology: Clinical and Experimental, 23(7), 571-585.

[6] Alibhai, F. J., Tsimakouridze, E. V., Reitz, C. J., Pyle, W. G., \& Martino, T. A. (2015). Consequences of circadian and sleep disturbances for the cardiovascular system. Canadian Journal of Cardiology, 31(7), 860872.

[7] Stevens, R. G. (2009). Light-at-night, circadian disruption and breast cancer: assessment of existing evidence. International journal of epidemiology, 38(4), 963-970.

[8] Kvaskoff, M., \& Weinstein, P. (2010). Are some melanomas caused by artificial light?. Medical hypotheses, 75(3), 305-311.

[9] Khademagha, P., Aries, M. B. C., Rosemann, A. L. P., \& van Loenen, E. J. (2016). Implementing nonimage-forming effects of light in the built environment: A review on what we need. Building and Environment, 108, 263-272.

[10] Visser, E. K., Beersma, D. G., \& Daan, S. (1999). Melatonin suppression by light in humans is maximal when the nasal part of the retina is illuminated. Journal of biological rhythms, 14(2), 116-121.

[11] Lasko, T. A., Kripke, D. F., \& Elliot, J. A. (1999). Melatonin suppression by illumination of upper and lower visual fields. Journal of biological rhythms, 14(2), 122-125.

[12] Glickman, G., Hanifin, J. P., Rollag, M. D., Wang, J., Cooper, H., \& Brainard, G. C. (2003). Inferior retinal light exposure is more effective than superior retinal exposure in suppressing melatonin in humans. Journal of biological rhythms, 18(1), 71-79.

[13] Rüger, M., Gordijn, M. C., Beersma, D. G., de Vries, B., \& Daan, S. (2005). Nasal versus temporal illumination of the human retina: effects on core body temperature, melatonin, and circadian phase. Journal of biological rhythms, 20(1), 60-70. 
[14] Dkhissi-Benyahya, O., Rieux, C., Hut, R. A., \& Cooper, H. M. (2006). Immunohistochemical evidence of a melanopsin cone in human retina. Investigative ophthalmology \& visual science, 47(4), 1636-1641.

[15] Do, M. T. H., \& Yau, K. W. (2010). Intrinsically photosensitive retinal ganglion cells. Physiological reviews, 90(4), 1547-1581.

[16] Sand, A., Schmidt, T. M., \& Kofuji, P. (2012). Diverse types of ganglion cell photoreceptors in the mammalian retina. Progress in retinal and eye research, 31(4), 287-302.

[17] Münch, M., \& Kawasaki, A. (2013). Intrinsically photosensitive retinal ganglion cells: classification, function and clinical implications. Current opinion in neurology, 26(1), 45-51.

[18] Rollag, M. D., Berson, D. M., \& Provencio, I. (2003). Melanopsin, ganglion-cell photoreceptors, and mammalian photoentrainment. Journal of biological rhythms, 18(3), 227-234.

[19] Zhao, X., Stafford, B. K., Godin, A. L., King, W. M., \& Wong, K. Y. (2014). Photoresponse diversity among the five types of intrinsically photosensitive retinal ganglion cells. The Journal of physiology, 592(7), 1619-1636.

[20] Galindo-Romero, C., Jiménez-López, M., Garcia-Ayuso, D., Salinas-Navarro, M., Nadal-Nicolás, F. M., Agudo-Barriuso, M., ... \& Vidal-Sanz, M. (2013). Number and spatial distribution of intrinsically photosensitive retinal ganglion cells in the adult albino rat. Experimental eye research, 108, 84-93.

[21] Nadal-Nicolás, F. M., Salinas-Navarro, M., Jiménez-López, M., Sobrado-Calvo, P., Villegas-Pérez, M. P., Vidal-Sanz, M., \& Agudo-Barriuso, M. (2014). Displaced retinal ganglion cells in albino and pigmented rats. Frontiers in neuroanatomy, 8, 99.

[22] Procyk, C. A., Eleftheriou, C. G., Storchi, R., Allen, A. E., Milosavljevic, N., Brown, T. M., \& Lucas, R. J. (2015). Spatial receptive fields in the retina and dorsal lateral geniculate nucleus of mice lacking rods and cones. Journal of neurophysiology, 114(2), 1321-1330.

[23] Ecker, J. L., Dumitrescu, O. N., Wong, K. Y., Alam, N. M., Chen, S. K., LeGates, T., ... \& Hattar, S. (2010). Melanopsin-expressing retinal ganglion-cell photoreceptors: cellular diversity and role in pattern vision. Neuron, 67(1), 49-60.

[24] Dacey, D. M., Liao, H. W., Peterson, B. B., Robinson, F. R., Smith, V. C., Pokorny, J., ... \& Gamlin, P. D. (2005). Melanopsin-expressing ganglion cells in primate retina signal colour and irradiance and project to the LGN. Nature, 433(7027), 749-754.

[25] Morin, L. P., Blanchard, J. H., \& Provencio, I. (2003). Retinal ganglion cell projections to the hamster suprachiasmatic nucleus, intergeniculate leaflet, and visual midbrain: bifurcation and melanopsin immunoreactivity. Journal of Comparative Neurology, 465(3), 401-416.

[26] Jusuf, P. R., Lee, S., Hannibal, J., \& Grünert, U. (2007). Characterization and synaptic connectivity of melanopsin-containing ganglion cells in the primate retina. European Journal of Neuroscience, 26(10), 29062921.

[27] Ingham, E. S., Günhan, E., Fuller, P. M., \& Fuller, C. A. (2009). Immunotoxin-induced ablation of melanopsin retinal ganglion cells in a non-murine mammalian model. Journal of Comparative Neurology, 516(2), 125-140.

[28] Li, Y., Li, C., Chen, Z., He, J., Tao, Z., \& Yin, Z. Q. (2012). A MicroRNA, mir133b, suppresses melanopsin expression mediated by failure dopaminergic amacrine cells in RCS rats. Cellular signalling, 24(3), 685-698.

[29] Esquiva, G., Lax, P., \& Cuenca, N. (2013). Impairment of Intrinsically Photosensitive Retinal Ganglion Cells Associated With Late Stages of Retinal DegenerationImpairment of ipRGCs in Retinal Degeneration. Investigative ophthalmology \& visual science, 54(7), 4605-4618.

[30] Hughes, S., Watson, T. S., Foster, R. G., Peirson, S. N., \& Hankins, M. W. (2013). Nonuniform distribution and spectral tuning of photosensitive retinal ganglion cells of the mouse retina. Current Biology, 23(17), 1696-1701.

[31] Nadal-Nicolás, F. M., Madeira, M. H., Salinas-Navarro, M., Jiménez-López, M., Galindo-Romero, C., Ortín-Martínez, A., ... \& Agudo-Barriuso, M. (2015). Transient Downregulation of Melanopsin Expression After Retrograde Tracing or Optic Nerve Injury in Adult RatsMelanopsin Expression After Axotomy or Tracing. Investigative ophthalmology \& visual science, 56(8), 4309-4323.

[32] Nadal-Nicolás, F. M., Sobrado-Calvo, P., Jiménez-López, M., Vidal-Sanz, M., \& Agudo-Barriuso, M. (2015). Long-Term Effect of Optic Nerve Axotomy on the Retinal Ganglion Cell LayerOptic Nerve Axotomy and Ganglion Cell Layer. Investigative ophthalmology \& visual science, 56(10), 6095-6112.

[33] Jeong, M. J., \& Jeon, C. J. (2015). Localization of melanopsin-immunoreactive cells in the Mongolian gerbil retina. Neuroscience research, 100, 6-16.

[34] Garcia-Ayuso, D., Di Pierdomenico, J., Esquiva, G., Manuel, F., \& Villegas-Pérez, M. P. (2015). Inherited photoreceptor degeneration causes the death of melanopsin positive retinal ganglion cells and increases their co-expression of Brn3a. Invest Ophthalmol Vis Sci, 56, 4592-4604.

[35] Semo, M. A., Coffey, P., Gias, C., \& Vugler, A. (2016). Retrograde Melanopsin Signaling Increases With Age in Retinal Degenerate Mice Lacking Rods and the Majority of Cones. Investigative ophthalmology \& visual science, 57(1), 115-25.

[36] Hannibal, J., Hindersson, P., Knudsen, S. M., Georg, B., \& Fahrenkrug, J. (2002). The photopigment melanopsin is exclusively present in pituitary adenylate cyclase-activating polypeptide-containing retinal ganglion cells of the retinohypothalamic tract. J Neurosci, 22(1), RC191. 
[37] Llamosas, M. M., Foster, R. G., \& Jeffery, G. (2005). Melanopsin (Opn4) positive cells in the cat retina are randomly distributed across the ganglion cell layer. Visual neuroscience, 22(1), 111.

[38] Semo, M. A., Lupi, D., Peirson, S. N., Butler, J. N., \& Foster, R. G. (2003). Light-induced c-fos in melanopsin retinal ganglion cells of young and aged rodless/coneless (rd/rd cl) mice. European Journal of Neuroscience, 18(11), 3007-3017. 